\title{
Antinociceptive, anti-inflammatory and antipyretic effects of solanum nigrum aqueous extract in animal models
}

\begin{abstract}
The present study was carried out to evaluate the antinociceptive, anti-inflammatory and antipyretic effects of the aqueous extract of Solanum nigrum leaves using various animal models. The extract, at concentrations of 10, 50 and 100\%, was prepared by soaking (1:20; w/v) air-dried powdered leaves $(20 \mathrm{~g})$ in distilled water $(\mathrm{dH} 2 \mathrm{O})$ for $72 \mathrm{~h}$. The extract solutions were administered subcutaneously in mice/rats $30 \mathrm{~min}$ prior to the tests. The extract exhibited significant $(\mathrm{P}<0.05)$ antinociceptive activity when assessed using the abdominal constriction, hot plate and formalin tests. The extract also produced significant $(\mathrm{P}<0.05)$ anti-inflammatory and antipyretic activities when assessed using the carrageenan-induced paw edema and brewer's yeast-induced pyrexia tests, respectively. Overall, these activities occurred in a concentration-dependent manner, except for the 50\% concentration of the extract, which was not effective in the abdominal constriction test. In conclusion, the present study demonstrated that $\mathrm{S}$. nigrum leaves possessed antinociceptive, anti-inflammatory and antipyretic effects and thus supported traditional claims of its medicinal uses.
\end{abstract}

Keyword: Anti-inflammatory; Antinociceptive; Antipyretic; Aqueous extract; Solanum nigrum 